\title{
Bending Rigidity and Gaussian Bending Stiffness of Single-Layered Graphene
}

\author{
Yujie Wei, ${ }^{* \dagger}$ Baoling Wang, ${ }^{\dagger}$ Jiangtao $\mathrm{Wu}^{\dagger}{ }^{\dagger}$ Ronggui Yang, ${ }^{*} \neq$ and Martin L. Dunn ${ }^{*} \ddagger$ \\ ${ }^{\dagger}$ LNM, Institute of Mechanics, Chinese Academy of Sciences, Beijing 100190, P. R. China \\ ${ }^{\ddagger}$ Department of Mechanical Engineering, University of Colorado, Boulder, Colorado 80309, United States
}

ABSTRACT: Bending rigidity and Gaussian bending stiffness are the two key parameters that govern the rippling of suspended graphene-an unavoidable phenomenon of twodimensional materials when subject to a thermal or mechanical field. A reliable determination about these two parameters is of significance for both the design and the manipulation of graphene morphology for engineering applications. By combining the density functional theory calculations of energies of fullerenes and single wall carbon nanotubes with the configurational energy of membranes determined by Helfrich Hamiltonian, we have designed a theoretical approach to accurately determine the bending rigidity and Gaussian bending stiffness of single-layered graphene. The bending rigidity and Gaussian bending stiffness of single-layered graphene are $1.44 \mathrm{eV}\left(2.31 \times 10^{-19} \mathrm{~N} \mathrm{~m}\right)$ and $-1.52 \mathrm{eV}\left(2.43 \times 10^{-19} \mathrm{~N} \mathrm{~m}\right)$, respectively. The bending rigidity is close to the experimental result. Interestingly, the bending stiffness of graphene is close to that of lipid bilayers of cells about $1-2 \mathrm{eV}$, which might mechanically justify biological applications of graphene.

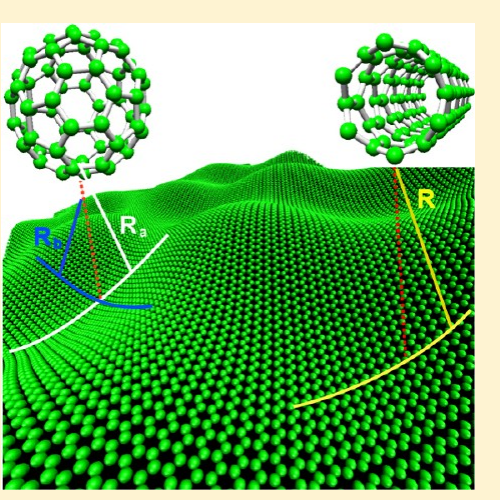

KEYWORDS: Bending rigidity, Gaussian bending stiffness, graphene, Helfrich Hamiltonian, density functional theory

$\mathrm{T}$ he mechanical properties of graphene ${ }^{1}$ are crucial to a variety of applications ranging from ultrafast electronics to nanocomposites and biological tissues. ${ }^{2-4}$ The unique combination of a high modulus $(\sim 1000 \mathrm{GPa})$ and tensile strength $(\sim 100 \mathrm{GPa})$, which are 1-2 orders of magnitude higher than those of commonly used metals such as stainless steel, and extremely small out-of-plane stiffness make such one atomic layer thick graphene an ideal candidate for biological membranes and stretchable electronics applications. ${ }^{4}$ The bending properties not only control the morphology of twodimensional graphene under external field stimuli, ${ }^{5-8}$ but also interplay with the electrical, magnetic, and thermal properties. ${ }^{1,9-16}$ Compared to the modulus and the tensile strength of graphene, its bending rigidity and Gaussian bending stiffness are much less studied. In this work, we have designed a theoretical approach to accurately determine the bending rigidity and Gaussian bending stiffness of single-layer graphene, by combining the density functional theory calculations with the Helfrich Hamiltonian for membranes.

There are always concerns about the applicability of the existing continuum mechanics theories for characterizing the mechanical properties of graphene or carbon nanotubes (CNTs). ${ }^{17}$ Instead of directly applying continuum mechanics theories to those low-dimensional carbon nanostructures, a combination of atomistic simulations with continuum theories is often used to deduce the mechanical properties of carbon nanostructures. ${ }^{18-21}$ Although there exist several studies for the bending stiffness of graphene, either based on the combination of continuum theory and molecular dynamics simulations with empirical potentials ${ }^{18-23}$ or by ab initio calculation, ${ }^{24-29}$ there lack efficient and accurate methods to simultaneously determine both the bending rigidity (normal bending stiffness) and the Gaussian bending stiffness of free-standing graphene. In this work, we apply the density functional theory (DFT) calculations to obtain the energies of zero-dimensional fullerenes and one-dimensional single-walled carbon nanotubes (SWCNTs) of different radii. We then derive both the bending rigidity and the Gaussian bending stiffness of free-standing single layered graphene, which are work-conjugate to the square of mean curvature and the square of Gaussian curvature, respectively.

For a membrane with a three-dimensional topology (see illustration in Figure 1), we can write the configurational energy $E$ described by the Helfrich Hamiltonian ${ }^{30,31}$ as:

$$
E=\int_{S}\left[\gamma+2 B_{M}\left(C_{M}-C_{0} / 2\right)^{2}+B_{G} C_{G}\right] d S
$$

where $\gamma$ is the energy for unitary flat surface, $B_{\mathrm{M}}$ is the bending rigidity, $B_{\mathrm{G}}$ is the Gaussian bending stiffness, $C_{\mathrm{M}}=\left(k_{1}+k_{2}\right) / 2$ is the mean curvature whereas $k_{1}$ and $k_{2}$ are the two principal curvatures of a three-dimensional surface, $C_{\mathrm{G}}=\left(k_{1} k_{2}\right)$ is the Gaussian curvature, and $C_{0}$ is spontaneous curvature, which disappears for symmetrical membranes. The integral in eq 1 extends over the whole surfaces. We can neglect $C_{0}$ without losing the accuracy in our following discussions due to the symmetry feature of fullerenes, SWCNTs, and graphene.

Received: August 26, 2012

Revised: November 14, 2012

Published: December 5, 2012 


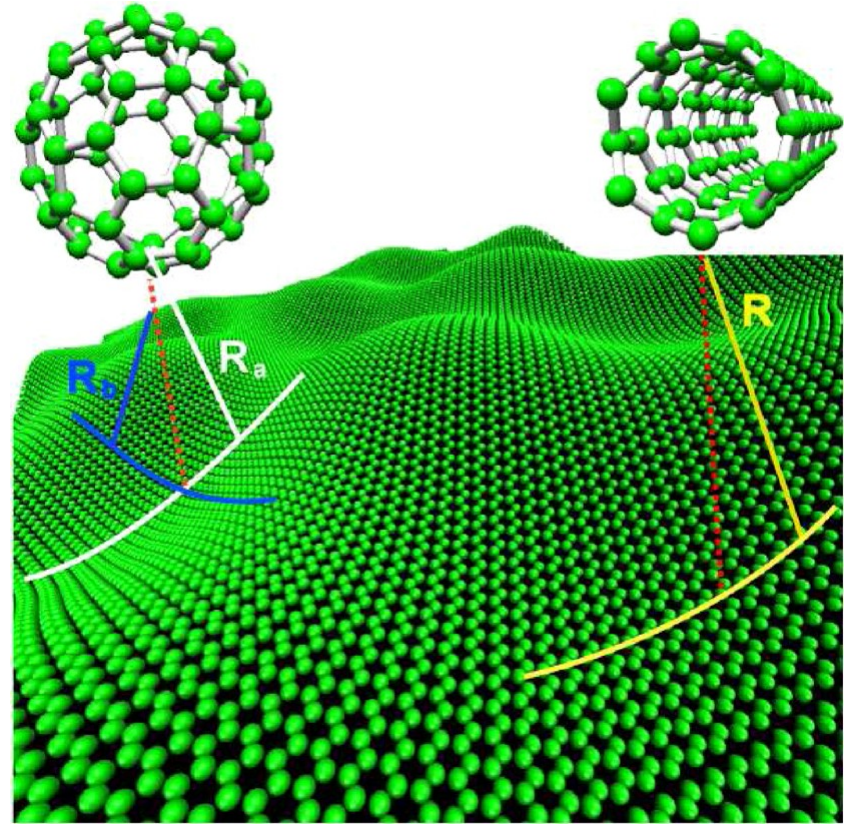

Figure 1. Illustration for the determination of the two elastic parameters controlling the corrugation of graphene. Given the geometrical nature of fullerenes and single wall carbon nanotubes, we need two curvature parameters $\left(k_{1}=1 / R_{\mathrm{a}}\right.$ and $\left.k_{2}=1 / R_{\mathrm{b}}\right)$ to describe the bending characteristics of a fullerene but one $(k=1 / R)$ for a CNT. This feature enables us to quantify the bending rigidity and Gaussian bending stiffness of graphene by adopting the Helfrich Hamiltonian.

By considering single layered graphene as a membrane, we assume that the surface energy $\gamma$ remains to be constant during pure bending. It has also been recognized that there may exist in-plane strain in SWCNTs ${ }^{19}$ and fullerenes. We have investigated the contribution of in-plane strain to the overall elastic energy in SWCNTs and fullerenes and found that the bending energy is at least one order-of-magnitude larger than that from in-plane deformation. This suggests the validity of eq 1 for samples considered here. By utilizing the fact that SWCNTs is a tube rolled up from a graphene, ${ }^{32}$ we can decouple $B_{\mathrm{M}}$ from $B_{\mathrm{G}}$ by considering infinitely long constantradius SWCNTs so that the term associated with the Gaussian curvature will disappear. By varying the radius of SWCNTs, we would then find that the energy per atom in SWCNT can be related to the energy per atom in graphene with the radii of SWCNTs by

$$
E_{\text {atom }}^{\mathrm{CNT}}=E_{0}+S_{0} B_{\mathrm{M}} r^{-2} / 2
$$

where $r$ is the radius of SWCNTs, $E_{\text {atom }}^{\mathrm{CNT}}$ is the energy per atom in a SWCNT, $E_{0}$ is the energy of an atom in a flat graphene, and $S_{0}=3 \sqrt{ } 3 d^{2} / 4=2.63 \AA^{2}$ is the planar footprint of a carbon atom in graphene with $d$ being the $\mathrm{C}-\mathrm{C}$ bond length. By calculating the energy per atom in SWCNTs with different radii, it is straightforward to derive $B_{\mathrm{M}}$, which is essentially the bending rigidity of graphene.

To determine the Gaussian bending stiffness $B_{\mathrm{G}}$, we consider a series of spheroidal fullerenes with total atoms from 60 to 540 , which have a corresponding radius variation from $1.86 \AA$ to about $11 \AA$. Using eq 1 , the energy per atom in fullerenes can be given as

$$
E_{\text {atom }}^{\mathrm{F}}=E_{0}+S_{0}\left(2 B_{\mathrm{M}}+B_{\mathrm{G}}\right) r^{-2}
$$

where $E_{\text {atom }}^{\mathrm{F}}$ is the energy per atom in a fullerene, and $r$ is the radius of a fullerene. In fullerene, the simplified average energy per atom shown in eq 3 , which is obtained by dividing the total energy of a fullerene by its number of atoms, includes contributions from both bending deformation and pentagon rings. To obtain $B_{\mathrm{G}}$ for intact graphene, we need to correct $E_{\text {atom }}^{\mathrm{F}}$ by excluding the extra energy due to the presence of 12 pentagon rings in a fullerene. Since the 12 pentagon rings involve 60 atoms, which is the size of $\mathrm{C}_{60}$, we will use the energy of $\mathrm{C}_{60}$ as a reference. For the total energy $E_{\text {total }}$ of a fullerene with more than 60 atoms, we subtract $E_{\text {total }}$ by the energy $\Delta E$ from the 60 atoms in 12 pentagons. The corrected energy per atom in the fullerene with more than 60 atoms is then obtained as

$$
E_{\text {atom }}^{\mathrm{F}}=\frac{E_{\text {total }}-\Delta E}{(N-60)}
$$

Now with $B_{\mathrm{M}}$ extracted using eq 2 from the calculation of SWCNTs, we could determine easily $B_{\mathrm{G}}$ by using eq 3 after obtaining $E_{\text {atom }}^{\mathrm{F}}$ for fullerenes of different radii. By using eq 4 , we assume that the energy in a fullerene can be additively attributed to pentagons and hexagons, which is supported by the highly localized deformation by pentagons in fullerenes. Experiments show that the bond lengths of the fullerenes are about $0.145 \pm 0.0015 \mathrm{~nm}$ for the bonds between pentagon and hexagon rings and $0.140 \pm 0.0015 \mathrm{~nm}$ for the bond between the hexagon rings. ${ }^{33}$ It suggests that the excessive energy by the
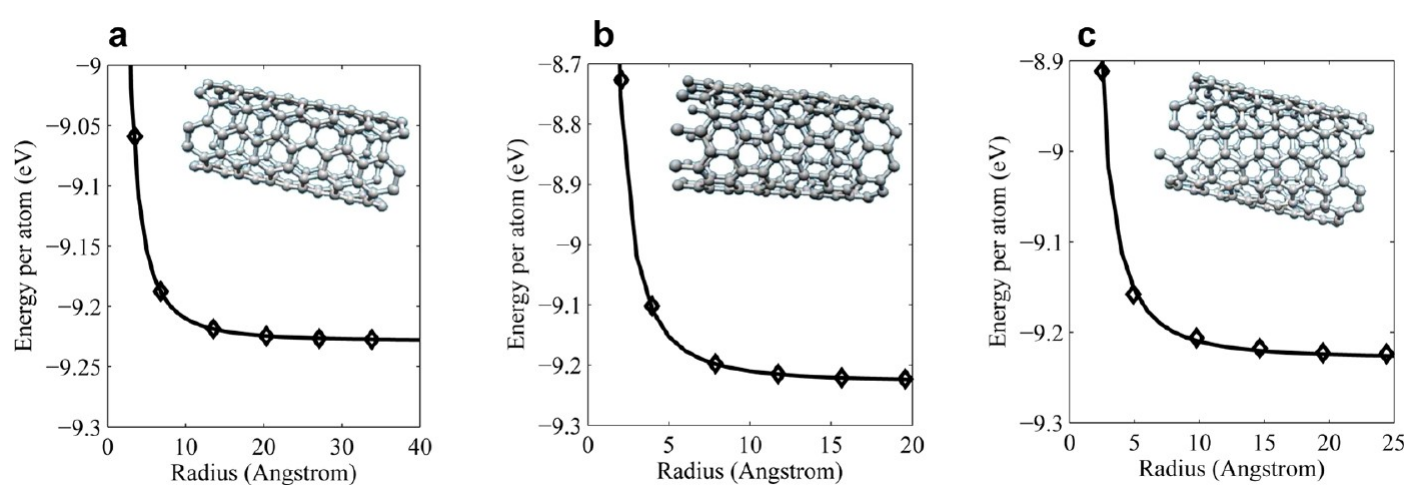

Figure 2. Energy per atom versus the radius in single-wall CNTs with different rolling up directions: DFT calculations (symbols) and continuum model (solid lines, with $B_{\mathrm{M}}=1.44 \mathrm{eV}$ ). (a) Rolled up along the armchair indices. (b) Rolled up along the zigzag indices. (c) Rolled up along the chiral indices $(n=5 i, m=2 i)$ for $i=1-6$. The insets in each figure show the typical tube structures. 
12 pentagons are highly localized and can be well-represented by the reference energy of $\mathrm{C}_{60}$.

We use DFT calculations to obtain the energies per carbon atom in SWCNTs and fullerenes. The calculations were performed using the Vienna Ab Initio Simulation Package (VASP) ${ }^{34,35}$ with the projector augmented wave (PAW) method and the Perdew-Burke-Ernzerhof (PBE) generalized gradient approximation (GGA) for the exchange and correlation terms. A plane-wave basis set with a kinetic energy cutoff of $400 \mathrm{eV}$ and a Monkhorst-Pack $k$-point mesh are used. Atoms are relaxed using a conjugate gradient algorithm until the interatomic forces are less than $0.03 \mathrm{eV} / \AA$.

For SWCNTs, the periodic boundary condition in the tube axis direction is applied. The vacuum space in nonperiodic directions (the radial direction) is set to be $1 \mathrm{~nm}$ to avoid interactions of SWCNTs or fullerenes with their periodic images. It is well-known that SWCNTs is commonly indexed by two integers $(n, m)$, from which the radius $r$ and the chiral angle $\chi$ can be deduced. ${ }^{32}$ As a reference, the energy per carbon atom in an infinite free-standing graphene (approximated by applying periodic boundary conditions in the length direction) is also calculated. In the absence of deformation, the $\mathrm{C}-\mathrm{C}$ bond length is calculated to be $d=0.1422 \mathrm{~nm}$ in the ideally flat graphene, which is in good agreement with the experimental value of $0.1421 \mathrm{~nm} .{ }^{32}$ The energy per atom in the ideally flat graphene is at $E_{0}=-9.229 \mathrm{eV}$.

Figure 2 shows the calculated energy per carbon atom in SWCNTs of different radii. Figure 2a shows the $E_{\text {atom }}^{\mathrm{CNT}}$ versus $r$ curve for SWCNTs with the tubes rolled up along the armchair direction (see the inset). The theoretical fitting to eq 2 renders the bending rigidity $B_{\mathrm{M}}=1.44 \mathrm{eV}$. This value is consistent with previous DFT calculations, as seen in Table 1 . Figure $2 b$ and $c$

Table 1. Bending Rigidity and Gaussian Bending Stiffness of Single-Layered Carbon Structures ${ }^{a}$

\begin{tabular}{|c|c|c|c|}
\hline authors [reference] & $B_{\mathrm{M}}(\mathrm{eV})$ & $B_{\mathrm{G}}(\mathrm{eV})$ & method \\
\hline this study & 1.44 & -1.52 & DFT \\
\hline Sanchez-Portal et al. ${ }^{26}$ & 1.52 & $\mathrm{~N} / \mathrm{A}$ & DFT \\
\hline Kudin et al. ${ }^{24}$ & $1.4-1.46$ & $\mathrm{~N} / \mathrm{A}$ & DFT \\
\hline Muñoz et al. ${ }^{29}$ & 1.48 & $\mathrm{~N} / \mathrm{A}$ & DFTB \\
\hline Koskinen and $\mathrm{Kit}^{28}$ & 1.61 & -0.7 & DFTB \\
\hline Tersoff $^{22}$ & 1.02 & $\mathrm{~N} / \mathrm{A}$ & empirical potential \\
\hline $\mathrm{Tu}$ and $\mathrm{Ou}-\mathrm{Yang}^{23}$ & 1.17 & N/A & empirical potential \\
\hline Arroyo and Belytschko ${ }^{27}$ & 0.83 & $\mathrm{~N} / \mathrm{A}$ & empirical potential \\
\hline Lu et al. ${ }^{19}$ & 1.4 & $\mathrm{~N} / \mathrm{A}$ & empirical potential \\
\hline Nicklow et al. ${ }^{39}$ & 1.2 & $\mathrm{~N} / \mathrm{A}$ & experiments \\
\hline
\end{tabular}

${ }^{a}$ Here DFTB is the abbreviation of density functional tight-binding model.

shows $E_{\text {atom }}^{\mathrm{CNT}}$ versus $r$ for SWCNTs rolled up along the zigzag and along the chiral indices of $(n=5 k, m=2 k$ where $k=1, \ldots$, $6)$, respectively. By fitting the numerical calculation results to eq 2, we essentially obtained the same value of bending rigidity $B_{\mathrm{M}}$ as that for the SWCNT rolled up along the armchair direction, which indicates that the bending rigidity is almost the same for SWCNTs with different chiralities. This result is indeed consistent with previous reports using DFT calculations, which showed that graphene is nearly isotropic at small strains, with Young's modulus $E=1050 \mathrm{GPa}$ and Poisson's ratio $v=$ $0.186^{24,25}$

Following the logic presented before, we can now determine the Gaussian bending stiffness of graphene by performing DFT calculations for fullerenes, after the calculation of the bending rigidity of graphene through DFT calculations of SWCNTs. Fullerenes form a spheroid shape with 12 pentagons and a variable number of hexagons. ${ }^{36-38}$ Table 2 gives the detailed

Table 2. Properties for Fullerenes of Different Radii Obtained by DFT Calculations ${ }^{a}$

$\begin{array}{ccrccc}\text { atoms }(N) & \text { K-point mesh } & E_{\text {total }}(\mathrm{eV}) & a \text {-axis }(\AA) & b \text {-axis }(\AA) & c \text {-axis }(\AA) \\ 60 & 2 \times 2 \times 2 & -531.33 & 3.335 & 3.419 & 3.475 \\ 70 & 2 \times 2 \times 2 & -622.58 & 3.465 & 3.542 & 3.973 \\ 76 & 2 \times 2 \times 2 & -676.39 & 3.357 & 3.831 & 4.381 \\ 78 & 2 \times 2 \times 2 & -693.86 & 3.576 & 3.679 & 4.298 \\ 80 & 2 \times 2 \times 2 & -711.77 & 3.864 & 3.929 & 4.056 \\ 84 & 2 \times 2 \times 2 & -748.46 & 3.277 & 4.250 & 4.791 \\ 100 & 2 \times 2 \times 2 & -894.22 & 3.881 & 4.052 & 5.635 \\ 180 & 2 \times 2 \times 2 & -1629.66 & 5.999 & 6.085 & 6.194 \\ 240 & 2 \times 2 \times 2 & -2181.51 & 6.958 & 6.941 & 7.241 \\ 320 & 1 \times 1 \times 1 & -2913.64 & 7.928 & 8.165 & 8.521 \\ 500 & 1 \times 1 \times 1 & -4569.75 & 9.902 & 10.247 & 10.853 \\ 540 & 1 \times 1 \times 1 & -4940.30 & 10.505 & 11.364 & 11.894\end{array}$

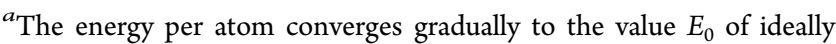
flat graphene.

information for fullerenes of different size after structure relaxation. Figure 3 gives the detailed geometries of several fullerenes including $\mathrm{C}_{60}, \mathrm{C}_{80}, \mathrm{C}_{180}, \mathrm{C}_{320}, \mathrm{C}_{500}$, and $\mathrm{C}_{540}$. For better viewing, atoms are colored based on their excessive energy in contrast to the energy of an atom in flat pristine graphene from corresponding molecular dynamics (MD) simulations. Here we have used MD package LAMMPS and adopted the AIREBO potential for carbon in our MD simulations. From the contour, we see that the excessive energy by the 12 pentagons are highly localized, which justifies the effectiveness when we apply eq 4. Clearly not all fullerenes are perfectly round. We have thus used the geometric mean of the three principal radii of a fullerene as the equivalent radius $r$. The principal radii are determined as follows. The $a$-axis is along the longest line connecting two pentagon poles in a fullerene. We could then determine the $c$-axis which is orthogonal to the $a$-axis but the shortest distance between the center and a point in the surface of the fullerene. The $b$-axis is found to be orthogonal to both $a$-axis and $c$-axis. The way to treat a fullerene as a sphere with the equivalent radius $r$ essentially stems from the Gauss-Bonner theorem, which states that the integral of Gaussian curvature over the closed compact oriented surface $M$ diffeomorphic to the sphere is equal to 2 . The difference between the area of a fullerenes and the approximation by using an equivalent sphere is normally less than $1 \%$ for fullerene with more than 100 atoms.

As discussed earlier for eq 4, we need to correct the energy per carbon atom in fullerenes before eq 3 can be successfully applied. Recall that $\mathrm{C}_{60}$ is the roundest one of all fullerenes, and each atom in a pentagon in $\mathrm{C}_{60}$ is shared by two hexagons, which is the case for all fullerenes with atomic number greater than 60 . Therefore, we use the energy per atom in $\mathrm{C}_{60}$ as the reference value for atoms in pentagons in fullerenes. As seen in Table $2, \Delta E=-531.33 \mathrm{eV}$ is then used in eq 4 for the calculation of energy $E_{\text {atom }}^{\mathrm{F}}$ per atom in all fullerenes with more than 60 atoms. Figure 4a shows the energy per atom as a function of the equivalent radii of fullerenes with more than 60 atoms from DFT calculations. With $B_{\mathrm{M}}=1.44 \mathrm{eV}$ from the fitting of Figure 2, the DFT calculation results can be well- 

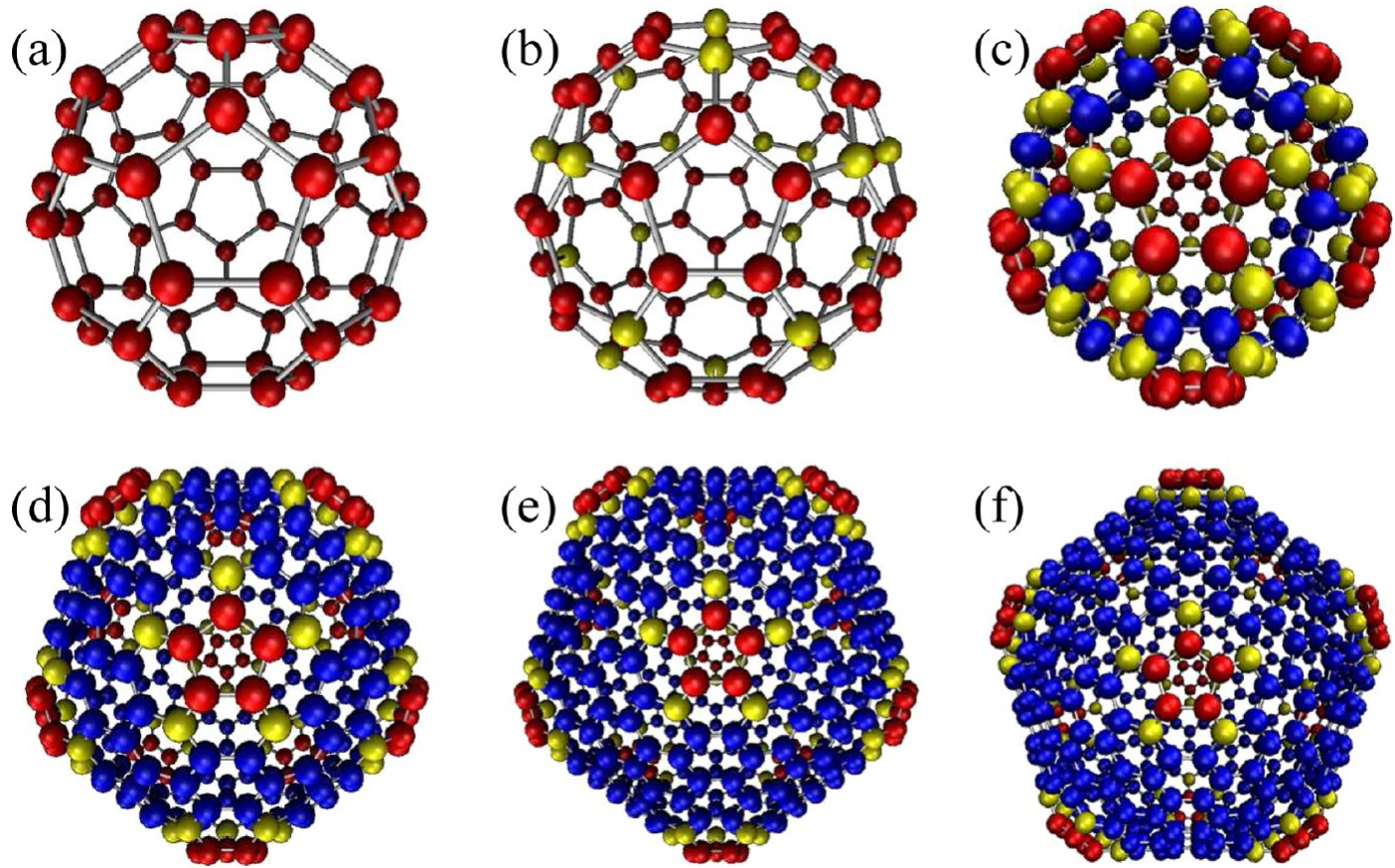

Figure 3. Energy contour from molecular dynamics simulations for fullerenes of different sizes. Atoms are colored based on the excessive energy in contrast to their reference energy state in flat graphene, with red, yellow, and blue, in turn, representing excessive energies about 0.60, 0.07, and 0.01 eV. (a) $\mathrm{C}_{60}$, (b) $\mathrm{C}_{80}$, (c) $\mathrm{C}_{180}$, (d) $\mathrm{C}_{320}$, (e) $\mathrm{C}_{500}$, and (f) $\mathrm{C}_{540}$. Note that each atom in a pentagon is shared by two other hexagons in fullerenes with an atom number greater than 60 .
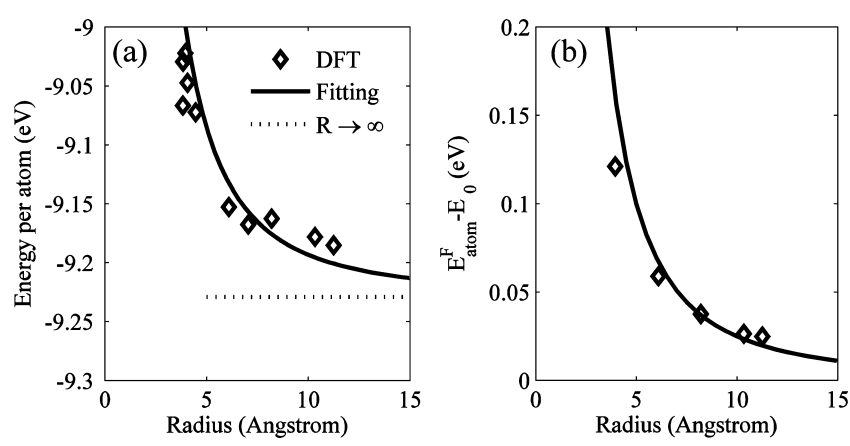

Figure 4. Energy per atom in fullerenes with different radii. (a) The results from DFT calculations (symbols), the fitted solid curve by the continuum model given in eq 3 , and the energy per atom for ideally flat graphene (dashed line) are shown. The DFT calculations can be well-captured by eq 3 by taking $B_{\mathrm{G}}=-1.52 \mathrm{eV}$. (b) The results from MD calculations (symbols) can be well-captured by eq 3 by using the same bending stiffness and Gaussian bending rigidity from DFT calculations.

captured with $B_{\mathrm{G}}=-1.52 \mathrm{eV}$ by fitting the results with eq 3 . As a comparison we list in Table 1 our simulation results and those from the literature. The results from our MD calculations are shown in Figure $4 \mathrm{~b}$. Since $E_{\text {atom }}^{\mathrm{F}}$ in a fullerene is accessible from $\mathrm{MD}$ simulations, we do not need to use eq 4 here. Now the energy per atom in a fullerene is an average over all atoms except the 60 atoms associated with all pentagons in the fullerene. $E_{\text {atom }}^{\mathrm{F}}-E_{0}$ from $\mathrm{MD}$ simulations can be welldescribed by eq 3 with the same bending stiffness and Gaussian bending rigidity from DFT calculations.

In contrast, the continuum mechanics-based Kirchhoff-Love theory for thin plates predicts $B_{\mathrm{M}}=22.3 \mathrm{eV}$ (with $B_{\mathrm{M}}=E H^{3}$ / $12\left(1-v^{2}\right), E=1050 \mathrm{GPa}$, and $\left.v=0.186^{26-28}\right)$ with $H=0.34$ $\mathrm{nm}$ used as the thickness of single layered graphene, which is one order-of-magnitude higher than the result reported here. This discrepancy indicates the breakdown of the applicability of continuum theory to predict bending properties in graphene. We note that there are discussions that $H$ may not be $0.34 \mathrm{~nm}$ for single layered graphene $e^{19,27,28}$ and a smaller effective thickness might reconcile the difference for $B_{M}$ between the prediction by continuum mechanics theory and that from DFT calculation. The bending stiffness of single layered graphene is very close to those numbers of the lipid bilayers in cells, ${ }^{31}$ which is about $1-2 \mathrm{eV}$. The parameters given here could be hence valuable for investigations about the interaction between the graphene and the cell member. ${ }^{40}$

In summary, by combining density functional theory calculations of carbon energies in the zero-dimensional fullerenes and one-dimensional single-walled carbon nanotubes, with the Helfrich Hamiltonian for the configurational energy of membranes, we determined the bending rigidity and Gaussian bending stiffness of single-layered graphene to be $1.44 \mathrm{eV}(2.31$ $\left.\times 10^{-19} \mathrm{~N} \mathrm{~m}\right)$ and $-1.52 \mathrm{eV}\left(2.43 \times 10^{-19} \mathrm{~N} \mathrm{~m}\right)$, respectively. These two elastic parameters of graphene are crucial for structure and morphology manipulation in the proposed biological and stretchable electronic applications.

\section{AUTHOR INFORMATION}

\section{Corresponding Author}

*E-mail: (Y.W.) yujie_wei@lnm.imech.ac.cn; (R.Y.) ronggui. yang@colorado.edu; and (M.D.) martin.dunn@colorado.edu.

Notes

The authors declare no competing financial interest.

\section{ACKNOWLEDGMENTS}

The authors acknowledge support from the Chinese Academy of Sciences and MOST 973 of China (No. 2012CB937500) and National Natural Science Foundation of China (NSFC) 
(11021262) (Y.J.W.) and AFOSR (Grant No. FA9550-11-10109) (R.G.Y.).

\section{REFERENCES}

(1) Novoselov, K. S.; Geim, A. K.; Morozov, S. V.; Jiang, D.; Zhang, Y.; Dubonos, S. V.; Grigorieva, I. V.; Firsov, A. A. Science 2004, 306, 666. Geim, A. K. Science 2009, 324, 1530.

(2) Iijima, S. Nature 1991, 354, 56.

(3) Wong, E. W.; Sheehan, P. E.; Lieber, C. M. Science 1997, 277, 1971.

(4) Rogers, J. A.; Lagally, M. G.; Nuzzo, R. G. Nature 2011, 477, 45.

(5) Yakobson, B. I.; Brabec, C. J.; Bernholc, J. Phys. Rev. Lett. 1996, 76, 2511.

(6) Liu, Y.; Yakobson, B. I. Nano Lett. 2010, 10, 2178.

(7) Yakobson, B. I.; Ding, F. ACS Nano 2011, 5, 1569.

(8) Fasolino, A.; Los, J. H.; Katsnelson, M. I. Nat. Mater. 2007, 6, 858.

(9) San-Jose, P.; Gonzalez, J.; Guinea, F. Phys. Rev. Lett. 2011, 106, 045502.

(10) Schebarchov, D.; Hendy, S. C.; Ertekin, E.; Grossman, J. C. Phys. Rev. Lett. 2011, 107, 185503.

(11) Guinea, F.; Geim, A. K.; Katsnelson, M. I.; Novoselov., K. S. Phys. Rev. B 2010, 81, 035408.

(12) Liu, P.; Zhang, Y. W. Appl. Phys. Lett. 2009, 94, 231912.

(13) Schniepp, H. C.; Kudin, K. N.; Li, J.-L.; Prud'homme, R. K.; Car, R.; Saville, D. A.; Aksay, I. A. ACS Nano 2008, 2, 2577.

(14) Cadelano, E.; Palla, P. L.; Giordano, S.; Colombo, L. Phys. Rev. B 2010, 82, 235414.

(15) Zhang, Z.; Liu, B.; Hwang, K. C.; Gao, H. Appl. Phys. Lett. 2011, 98, 121909.

(16) Li, X.; Maute, K.; Dunn, M. L.; Yang, R. G. Phys. Rev. B 2010, 81,245318

(17) Wei, Y.; Wu, J.; Yin, H.; Shi, X.; Yang, R. G.; Dresselhaus, M. S. Nat. Mater. 2012, 11, 759.

(18) Zhang, D. B.; Akatyeva, E.; Dumitrică, T. Phys. Rev. Lett. 2011, 106, 255503.

(19) Lu, Q.; Arroyo, M.; Huang, R. J. Phys. D: Appl. Phys. 2009, 42, 102002.

(20) Cadelano, E.; Palla, P. L.; Giordano, S.; Colombo, L. Phys. Rev. Lett. 2009, 102, 235502.

(21) Peng, J.; Wu, J.; Huang, K. C.; Song, J.; Huang, Y. J. Mech. Phys. Solids 2008, 56, 2213.

(22) Tersoff, J. Phys. Rev. B 1992, 46, 15546.

(23) Tu, Z. C.; Ou-Yang, Z. C. Phys. Rev. B 2002, 65, 233407.

(24) Kudin, K. N.; Scuseria, G. E.; Yakobson, B. I. Phys. Rev. B 2001, 64, 235406.

(25) Liu, F.; Ming, P.; Li, J. Phys. Rev. B 2007, 76, 064120.

(26) Sanchez-Portal, D.; Artacho, E.; Soler, J. M.; Rubio, A.; Ordejon, P. Phys. Rev. B 1999, 59, 12678.

(27) Arroyo, M.; Belytschko, T. Phys. Rev. B 2004, 69, 115415.

(28) Koskinen, P.; Kit, O. O. Phys. Rev. B 2010, 82, 235420.

(29) Muñoz, E.; Singh, A. K.; Ribas, M. A.; Penev, E. S.; Yakobson, B. I. Diamond Relat. Mater. 2010, 19, 368.

(30) Helfrich, W. Z. Naturforsch. C 1973, 28, 693.

(31) Lipowsky, R. Nature 1991, 349, 475.

(32) Saito, R.; Fujita, M.; Dresselhaus, G.; Dresselhaus, M. S. Physical Properties of Carbon Nanotubes; Imperial College London: London, 1998.

(33) Jonhson, R. D.; Bethune, D. S.; Yannoni, C. S. Acc. Chem. Rec. 1992, 25, 169.

(34) Kresse, G.; Furthmüller, J. Phys. Rev. B 1996, 54, 11169.

(35) Kresse, G.; Furthmüller, J. Comput. Mater. Sci. 1996, 6, 15.

(36) Krato, H. W.; McKay, K. Nature 1988, 331, 328.

(37) Smalley, R. E. Acc. Chem. Res. 1992, $25,98$.

(38) Diederich, F.; Whetten, R. L. Acc. Chem. Res. 1992, 25, 119.

(39) Nicklow, R.; Wakabayashi, N.; Smith, H. G. Phys. Rev. B 1972, 5, 4951.

(40) Frost, R.; Jönsson, G. E.; Chakarov, D.; Svedhem, S.; Kasemo, B. Nano Lett. 2012, 12, 3356. 